\title{
Autotuning for delay systems using meromorphic functions
}

\author{
R. Prokop, L. Pekař, J. Korbel \\ Tomas Bata University in Zlin, Faculty of Applied Informatics \\ Nad Stráněmi 4511, 76005 Zlín, Czech Republic. \\ (tel: +420 57603 5257; e-mail:pekar@fai.utb.cz,prokop@fai.utb.cz)
}

\begin{abstract}
The paper presents an autotuning method for time delay systems. The novelty in principles is a new combination of biased-relay feedback identification and an algebraic control design method for timedelay systems. The estimation of the controlled process is based on an asymmetrical limit cycle data experiment. Then, a stable transfer function with a dead-time term is identified. The controller is designed through solutions of Diophantine equations in the ring of stable and proper retarded quasipolynomial meromorphic functions. Controller parameters are tuned through a pole-placement problem as a desired multiple root of the characteristic closed loop equation. First and second order identification gives Smith-like feedback controllers with the realistic PI and PID structure. The design principle also offers a scalar tuning parameter $\mathrm{m}_{0}>0$ which can be adjusted by a suitable principle or an optimization method. The developed approach is illustrated by examples in the Matlab + Simulink environment.
\end{abstract}

Keywords: Relay feedback test, Limit cycles, Delay, Algebraic approaches, Equalization method.

\section{INTRODUCTION}

Time delay systems constitute an indispensable family of industrial processes. A feedback loop is the most efficient manner how to change system properties. However, thanks to the feedback loop, time delay notably affects whole system dynamics. During recent decades various approaches and algorithms have been researched for compensating the influence of time delay in a feedback loop. In addition to that, many control design principles to obtain satisfactory loop behavior have been presented. There surely exist several classifications of control design methods for time delay systems. Nowadays, three main groups dominate. The first group contains approaches based on Smith predictor structure, or more precisely its modifications, see Smith (1957), Majhi and Atherton (1998), Kaya and Atherton (1999). These methods assume model of the controlled system in feedback loops, thus, it pertains into IMC (Internal Model Controllers). Second group consists of predictive based approaches, mainly using state-space description, Fliess et al. (2002). Last but not least, third group of algebraic approaches is assumed, Kučera (1993), Prokop and Corriou (1997), Prokop et al. (2002). Just a method from the third group is described in the paper.

Automatic tuning became a very desirable feature in industrial applications as well as in control engineers. Nowadays, there are many different auto-tuning principles, Aström and Hägglund (1984), Pecharromán and Pagola (2000), Arruda and Barros (2001), Thyagarajan and Yu (2002). An auto-tuning procedure consists of a process identification experiment plus a controller design method. The present day trend is a relay feedback test. During the first step, a feedback relay test is performed and utilized for estimation. The controlled estimated system can be identified through the ultimate parameters, Aström and Hägglund (1984), or by the transfer function estimation, Pecharromán and Pagola (2000), Vítečková and Víteček (2004). The second step represents a consecutive control design procedure which can have various modifications for stable, unstable or delayed systems.

This contribution brings a novel combination of an identification test based on a biased relay with hysteresis and an algebraic control design utilizing Diophantine equation solutions in a special $R_{M S}$ ring. First and second order model with one time constant and time delay is assumed as examples for control applications giving a class of a PI or PID like controllers with a Smith predictor structure.

\section{DESCRIPTION AND CONTROL DESIGN OF TIME DELAY SYSTEMS}

Modern control theory frequently prefers algebraic parlance and tools like rings and linear equations. There are several rings, e.g. the ring of polynomials $R_{P}$, the ring of stable and proper rational function $R_{P S}$ etc., see Kučera (1993), Prokop and Corriou (1997), Vidyasagar (1985), which can be used for control syntheses. Different rings require various approximations of delay terms which reduce quality of a model. The most known is the Pade approximation, respecting the relative degree of the original transfer function. As a consecution, final control design obviously gives controllers of quite high degrees. This paper utilizes a ring of stable and proper meromorphic functions $R_{M S}$ omitting any approximation which was developed especially for delay systems, Zítek and Kučera (2003). 
An element of this ring is a ratio of two retarded quasipolynomials $y(\mathrm{~s}) / x(\mathrm{~s})$. A retarded quasipolynomial $x(s)$ of degree $n$ means

$$
x(s)=s^{n}+\sum_{i=0}^{n-1} \sum_{j=1}^{h} x_{i j} s^{i} \exp \left(-\vartheta_{i j} s\right), \vartheta_{i j} \geq 0
$$

where retarded refers to the fact that the highest $s$-power is not affected by exponentials. A more general notion called neutral quasipolynomials also can be used in this sense, see Pekař end Prokop (2009). Quasipolynomial (1) is stable when it owns no finite zero $s_{0}$ such that $\operatorname{Re}\left\{s_{0}\right\} \geq 0$. For stability tests, see e.g. in Zítek and Kučera (2003), Zítek and Víteček (1999).

The denominator of the ratio in $R_{M S}$ is supposed to be stable, while the numerator $y(s)$ of an element in $R_{M S}$ can be factorized in the form $y(s)=\tilde{y}(s) \exp (-\tau s)$, where $\tau \geq 0$ and $\tilde{y}(s)$ is any retarded quasipolynomial. The ratio $y(\mathrm{~s}) / x(\mathrm{~s})$ is called proper when the degree of the numerator is less or equal to the degree of the denominator.

A linear time-invariant delay system then can be expressed as a ratio of two elements of the $R_{M S}$ ring. An example of the first order system with input-output time delay is then

$$
\begin{aligned}
& G(s)=\frac{\frac{K \exp (-\tau s)}{s+m_{0} \exp \left(-\vartheta_{s}\right)}}{\frac{T s+1}{s+m_{0} \exp \left(-\vartheta_{s}\right)}}=\frac{\frac{b(s)}{m(s)}}{\frac{a(s)}{m(s)}}=\frac{B(s)}{A(s)} \\
& A(s), B(s) \in R_{M S}, m_{0}>0
\end{aligned}
$$

The structure for control design suggested below is assumed in a classical feedback loop displayed in Fig. 1.

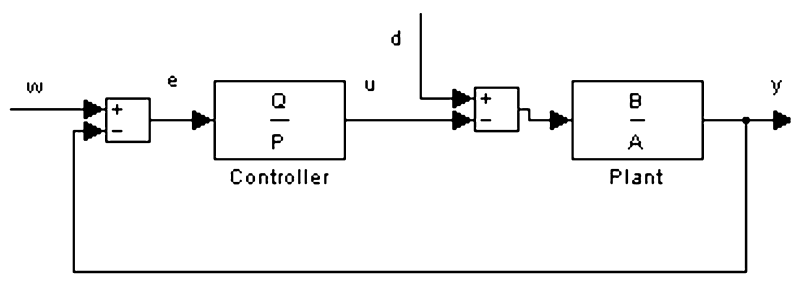

Fig. 1. Feedback control loop

Let a model transfer function be expressed as

$G(s)=\frac{B(s)}{A(s)}, A(s), B(s) \in R_{M S}$

and a controller be of a structure

$G_{R}(s)=\frac{Q(s)}{P(s)}, Q(s), P(s) \in R_{M S}$

In a similar way, reference and load disturbance signals are expressed by

$$
\begin{aligned}
& W(s)=\frac{H_{W}(s)}{F_{W}(s)}, H_{W}(s), F_{W}(s) \in R_{M S} \\
& D(s)=\frac{H_{D}(s)}{F_{D}(s)}, H_{D}(s), F_{D}(s) \in R_{M S}
\end{aligned}
$$

The aim of the control synthesis is to (internally) stabilize the feedback control system with asymptotic tracking and load disturbance attenuation.

The first step of the stabilization can be formulated in an elegant way in $R_{M S}$ by the Diophantine equation

$$
A(s) P_{0}(s)+B(s) Q_{0}(s)=1
$$

where $P_{0}(s)$ a $Q_{0}(s)$ is a particular solution from $R_{M S}$. Since for stable systems, the $R_{M S}$ ring constitutes the Bézout domain, Pekař and Prokop (2009), the solution of (7) always exists. All stabilizing controllers can be expressed in a parametric form by

$$
\begin{aligned}
& \frac{Q(s)}{P(s)}=\frac{Q_{0}(s)+A(s) Z(s)}{P_{0}(s)-B(s) Z(s)} \\
& P_{0}(s)-B(s) Z(s) \neq 0
\end{aligned}
$$

where $Z(s)$ is an arbitrary element of $R_{M S}$. The special choice of this element can ensure additional control conditions. Details and proofs can be found e.g. in Kučera (1993), Prokop and Corriou (1997), Prokop et al. (2002), Vidyasagar (1985).

Asymptotic tracking and disturbance attenuation result from expression for $E(s)$ which reads

$$
E(s)=\frac{A(s) P(s)}{A(s) P(s)+B(s) Q(s)} W(s)-\frac{B(s) P(s)}{A(s) P(s)+B(s) Q(s)} D(s)
$$

and they lead to the condition that both $F_{w}(s)$ and $F_{D}(s)$ divide $P(s)$. Details about divisibility in $R_{M S}$ can be found, e.g. in Kučera (1993), Zítek and Kučera (2003).

\section{RELAY FEEDBACK TESTS}

An auto-tuning procedure consists of a process identification experiment plus a controller design method. The traditional method was proposed by Åström and Hägglund (1984), based on a symmetrical relay feedback test when a relay of magnitude $h_{r}$ is inserted in the feedback loop (see Fig. 2).

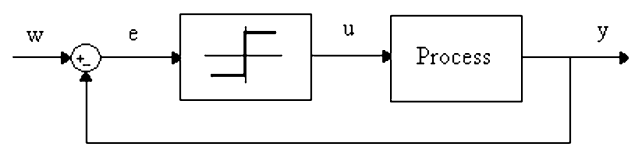

Fig. 2. Relay based identification

The goal of the original test was to indicate the critical point in the Nyquist curve of the open loop, see Aström and Hägglund (1984), Yu (1999). However, there are other relays used in identification experiments, e.g. the biased (asymmetrical) relay, a typical process response of which is depicted in Fig. 3.

It is well known that many stable industrial processes can be adequately approximated by model

$\widetilde{G}(s)=\frac{K \exp (-\tau s)}{T s+1}$ 
The gain $K$ of the process using a biased relay can be estimated by, Vyhlídal (2000)

$$
K=\int_{0}^{i T_{u}} y(t) d t / \int_{0}^{i T_{u}} u(t) d t ; i=1,2,3, \ldots
$$

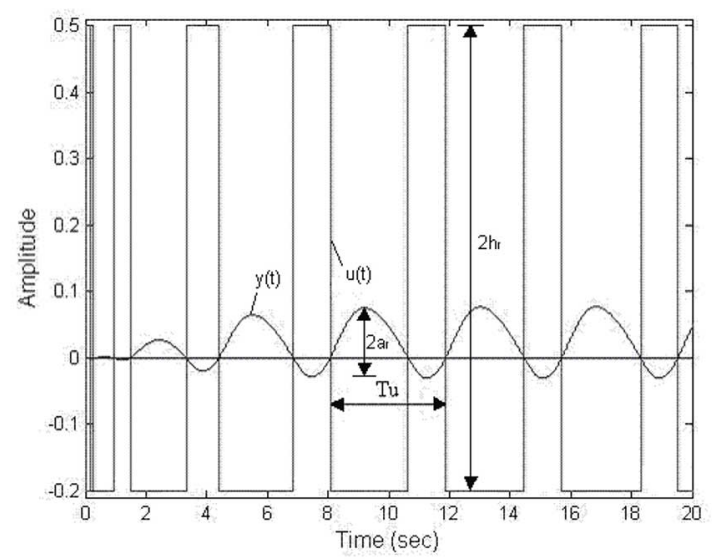

Fig. 3. Biased relay oscillation of stable processes.

Time constant $T$ is given by the analysis of limit cycles as

$$
T=\frac{T_{u}}{2 \pi} \cdot \sqrt{\frac{16 \cdot K^{2} \cdot h_{r}^{2}}{\pi^{2} \cdot a_{r}^{2}}-1}
$$

Methodology in Vítečková and Víteček (2004), Yu (1999) using a relay with hysteresis $\varepsilon$ also enables to estimate a delay term $\tau$ in (10) by

$$
\tau=\frac{T_{u}}{2 \pi}\left[\pi-\arctan \frac{2 \pi T}{T_{u}}-\arctan \frac{\varepsilon}{\sqrt{a_{r}{ }^{2}-\varepsilon^{2}}}\right]
$$

This relay can be also used for the estimation of the second order model with time delay

$$
\widetilde{G}(s)=\frac{K \exp (-\tau s)}{(T s+1)^{2}}
$$

where the gain $K$ is calculated by (11), and for $T$ and $\tau$ follows

$$
\begin{aligned}
& T=\frac{T_{u}}{2 \pi} \cdot \sqrt{\frac{4 \cdot K \cdot h_{r}}{\pi \cdot a_{r}}-1} \\
& \tau=\frac{T_{u}}{2 \pi}\left[\pi-2 \arctan \frac{2 \pi T}{T_{u}}-\arctan \frac{\varepsilon}{\sqrt{a_{r}^{2}-\varepsilon^{2}}}\right]
\end{aligned}
$$

After the relay feedback experiment, the estimated parameters $K$, $T$ and $\tau$ are used directly to calculate controller parameters.

\section{AUTOTUNING STRUCTURES AND TUNING}

\subsection{First order model}

Let the controlled process be described by a first order delayed model (10) where parameters $K, T$ and $\tau$ are estimated via relay identification test (11) - (13). The model coprime factorization in the $R_{M S}$ ring can be done e.g. as

$$
\widetilde{G}(s)=\frac{\frac{K \exp (-\tau s)}{s+m_{0}}}{\frac{T s+1}{s+m_{0}}}=\frac{B(s)}{A(s)}=\widetilde{G}_{1}(s)
$$

where $m_{0}>0$ is a free (selectable) scalar parameter.

The control loop is considered as a simple feedback system (Fig. 1) with controller (4) and both external inputs are step functions. Follow the algebraic controller design presented in Section 2.

The stabilizing Diophantine equation (7) reads

$$
\frac{T s+1}{s+m_{0}} P_{0}(s)+\frac{K \exp (-\tau s)}{s+m_{0}} Q_{0}(s)=1
$$

Choose $Q_{0}(s)=1$ which yields

$P_{0}(s)=\frac{s+m_{0}-K \exp (-\tau s)}{T s+1}$

Obviously, this solution does not satisfy the requirements of asymptotical reference tracking and disturbance attenuation, since $P_{0}(0) \neq 0$, thus, the particular solution ought to be parameterized as

$$
\begin{aligned}
P(s) & =P_{0}(s)-B(s) Z(s) \\
& =\frac{s+m_{0}-K \exp (-\tau s)}{T s+1}-\frac{K \exp (-\tau s)}{s+m_{0}} Z(s)
\end{aligned}
$$

In order to have $P(s)$ in a simple form satisfying $P_{0}(0)=0$, choose

$Z(s)=\frac{s+m_{0}}{T s+1}\left(\frac{m_{0}}{K}-1\right)$

which gives

$P(s)=\frac{s+m_{0}(1-\exp (-\tau s))}{T s+1}$

and contemporaneously

$Q(s)=\frac{m_{0}}{K}$

according to (8). Thus, the final anisochronic controller structure reads

$G_{R}(s)=\frac{m_{0}}{K} \frac{T s+1}{s+m_{0}(1-\exp (-\tau s))}=G_{R 1}(s)$ 
where $m_{0}$ serves as a tuning parameter. The denominator in (24) has infinite number of poles. The construction of this controller is more complex than usual PI or PID controllers.

\subsection{Second order model}

Now let a plant model be of the form (14) which can be formulated in $R_{M S}$ as

$$
\widetilde{G}(s)=\frac{\frac{K \exp (-\tau s)}{\left(s+m_{0}\right)^{2}}}{\frac{(T s+1)^{2}}{\left(s+m_{0}\right)^{2}}}=\frac{B(s)}{A(s)}=\widetilde{G}_{2}(s)
$$

Similarly as in (18) and (19) for a first order model, stabilizing particular solution of (7) is obtained as

$$
Q_{0}(s)=1, P_{0}(s)=\frac{\left(s+m_{0}\right)^{2}-K \exp (-\tau s)}{(T s+1)^{2}}
$$

and the parameterization (8) enables to satisfy the reference tracking and disturbance attenuation; hence the option

$$
Z(s)=\frac{\left(s+m_{0}\right)^{2}}{(T s+1)^{2}}\left(\frac{m_{0}^{2}}{K}-1\right)
$$

results in

$$
\begin{aligned}
& P(s)=\frac{s^{2}+2 m_{0} s+m_{0}^{2}(1-\exp (-\tau s))}{(T s+1)^{2}} \\
& Q(s)=\frac{m_{0}^{2}}{K}
\end{aligned}
$$

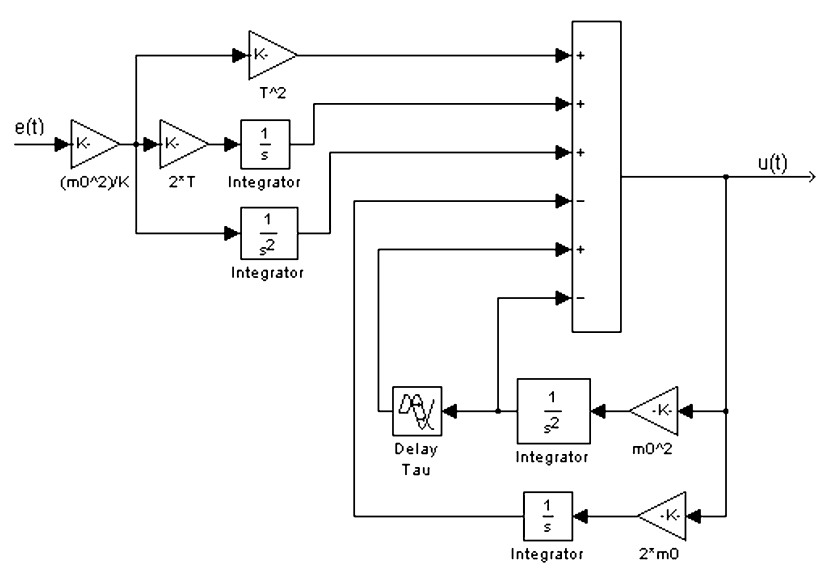

Fig. 4. Matlab-Simulink scheme of controller (30)

The controller structure is then

$$
G_{R}(s)=\frac{m_{0}^{2}}{K} \frac{(T s+1)^{2}}{s^{2}+2 m_{0} s+m_{0}^{2}(1-\exp (-\tau s))}=G_{R 2}(s)
$$

In order to demonstrate the anisochronic structure of the controller, its Matlab-Simulink scheme is depicted in Fig. 4.

\subsection{Controllers tuning}

The suitable choice of $m_{0}$ can be an effective and simple tuning tool since this real number strongly influenced all controller parameters and the characteristic (quasi)polynomial of the closed loop. The question of the right and/or optimal choice of $m_{0}$ has not been solved yet, although many possible attempts have been studied. An interesting and simple tuning principle of classical PI controllers was developed in Gorez and Klán (2000) called "equalization" method. The proposed convenient relations Parameters of controllers (24), (29) can be also tuned after some approximations of transfer functions (24), (29), see Pekař et al. (2007) for details.

The "equalization" tuning principle yields relations between PI controller and first order process parameters

$K_{P}=\frac{1}{K} \frac{1+(1-\vartheta)^{2}}{2}, T_{I}=(T+\tau) \frac{1+(1-\vartheta)^{2}}{2}$

where the normalized time delay given by $\vartheta=\tau /(T+\tau)$.

Consider the first order case and try to utilize identities (30) to the approximated controller structure. Obviously, the right hand side formula in (30) is fully accomplished if $\vartheta=0$. On the contrary, the left hand side formula leads to the requirement

$m_{0}=\frac{1}{T} \frac{1+(1-\vartheta)^{2}}{2}$

(28) Second, for the second order case, if the relation for $K_{P}$ in (30) is adopted for the PI part of approximated PID controller (29), then

$m_{0}=\frac{1}{T} \frac{3+2(1-\vartheta)^{2}}{4}$

By contrast, if the relation for $T_{I}$ in (30) is satisfied, then one can write

$$
m_{0}=\frac{1}{4 T-(T+\tau)\left[1+(1+\vartheta)^{2}\right]}
$$

As can be seen, equation (33) is not suitable for the controller design, because of the possibility to obtain a negative value of $m_{0}$ resulting in an unstable feedback response. On the other hand, equation (32) gives a reasonable choice for free parameter $m_{0}$, and consequently, following relations for controller parameters are derived

$$
T_{I}=\frac{4\left[1+(1-\vartheta)^{2}\right]}{3+2(1-\vartheta)^{2}} T, T_{D}=\frac{\left[3+2(1-\vartheta)^{2}\right]^{2}-8\left[1+(1-\vartheta)^{2}\right]}{4\left[3+2(1-\vartheta)^{2}\left[1+(1-\vartheta)^{2}\right]\right.} T(34)
$$

On the other hand, if (33) is satisfied, (34) implies 
$K_{P}=\frac{(T+\tau)\left[1+(1-\vartheta)^{2}\right]}{4 K\left[4 T-(T+\tau)\left[1+2(1-\vartheta)^{2}\right]\right.}$

$T_{D}=\frac{\left[(T+\tau)\left[1+(1-\vartheta)^{2}\right]-2 T\right]^{2}}{2(T+\tau)\left[1+(1-\vartheta)^{2}\right]}$

Relation for $T_{D}$ in (35) shows an interesting property. A case when $\tau=0$ leads to $T_{D}=0$, in other words, PI-like controller is obtained.

\section{PROGRAM IMPLEMENTATION AND SIMULATIONS}

A program system for design, tuning and simulation of introduced autotuning and control method was developed in the Matlab-Simulink environment. The Main menu of this program can be seen in Fig. 5. The program system is designed in a user-friendly philosophy.

Initially, the controlled transfer function is defined and parameters for the relay experiment must be entered. Then, the experiment is performed and it can be repeated with modified parameters if necessary. After the experiment, parameters of the estimated transfer function are calculated automatically and controller parameters are generated after pushing of the appropriate button. During the simulation routine, a standard Simulink scheme is performed and required outputs are displayed. The simulation horizon can be prescribed as well as tuning parameter $m_{0}$, other simulation parameters can be specified in the Simulink environment. In all simulation a change of the step reference is performed in the second third of the simulation horizon and a step change in the load is injected in the last third.

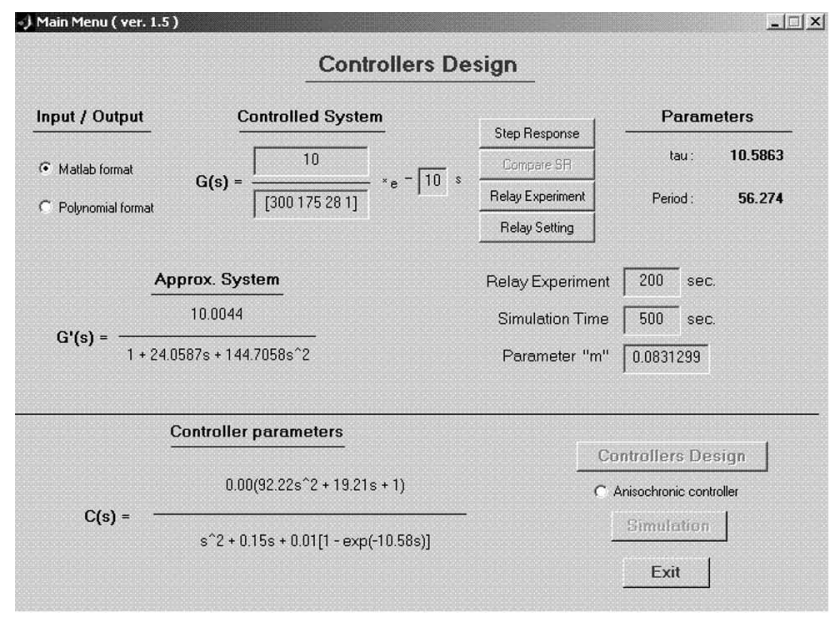

Fig. 5. Main menu of program system

As an example, a stable system with time delay governed by the transfer function of the third order was chosen

$G(s)=\frac{10 \exp (-10 s)}{(20 s+1)(5 s+1)(3 s+1)}$
A wide class of frequent stable industrial processes can be approximated by this type of transfer functions. The estimation was performed by the relay feedback experiment where asymmetric relay with hysteresis was used with adjusted parameters: $h_{r}=0.225(0.2$ when on, -0.25 when off), $\varepsilon=0.05$. Limit cycles result in $a_{r}=1.022, T_{u}=56.28$.

The first order model (10) using (11)-(13) gave

$\widetilde{G}_{1}(s)=\frac{10 \exp (-16.91 s)}{23.45 s+1}$

Relations (15) and (16) yields the second order model (14)

$\widetilde{G}_{2}(s)=\frac{10}{(12.03 s+1)^{2}} \exp (-11.04 s)$

The step responses of (36)-(38) are compared in Fig. 6. According to the mentioned algebraic control design in the $R_{M S}$ ring, the following controller was obtained through (18) -(24) in the form

$G_{R 1}(s)=2.85 \cdot 10^{-3} \frac{23.45 s+1}{s+2.85 \cdot 10^{-2}(1-\exp (-16.91 s))}$

where parameter $m_{0}=2.85 \cdot 10^{-2}$ was tuned by the “equalization" principle (31).

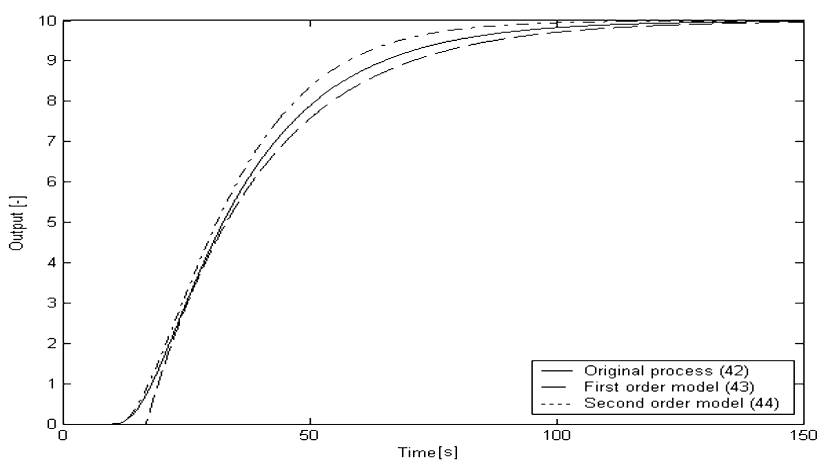

Fig. 6. Step responses of (36)-(38)

Controller (29) for the second order model is

$G_{R 2}(s)=\frac{5.43 \cdot 10^{-4}(12.03 s+1)^{2}}{s^{2}+14.74 \cdot 10^{-2} s+5.43 \cdot 10^{-3}(1-\exp (-11.04 s))}$

The parameter $m_{0}=7.37 \cdot 10^{-2}$ was calculated by (32) to satisfy $K_{P}$ in (30). Alternatively, keeping $T_{I}$ in (30) with respecting (33) results in $m_{0}=-0.04$ which is not acceptable.

Control responses for both models and controllers are compared in Fig. 7 (control actions) and in Fig. 8 (controlled variables). Reference signal is $w(t)=1$ for $t \in[0,200)$ and $w(t)=2$ for $t \in[200,600]$. Step input disturbance $d(t)=-0.1$ enters at $t=400 \mathrm{~s}$.

Control responses are rather slow; however, without abrupt changes of control signals (except instants of step changes of the reference signal). This result agrees with the philosophy 
of the "equalization" method which suggests a compromise between a suitable control response and carefulness to actuators. Generally, higher $m_{0}$ gives faster but more oscillating control responses, and vice-versa.

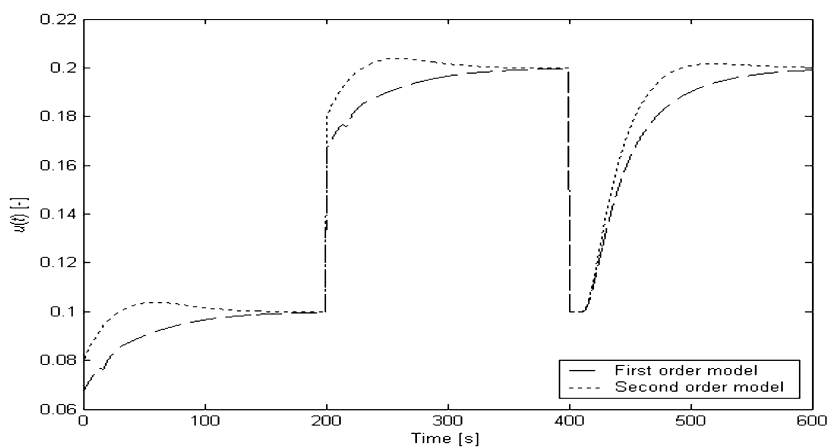

Fig. 7. Variable $u(t)$ of plant (36) controlled by (39) and (40).

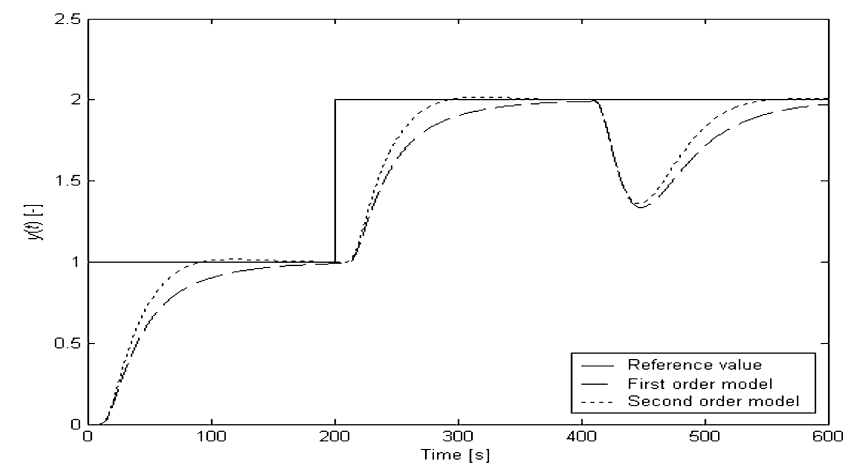

Fig. 8. Controlled output $y(t)$ of plant (36) using (39) and (40).

\section{CONCLUSIONS}

The contribution gives a new combination of relay feedback estimation and a control design method. A first and a second order models with time delay are used to estimate plant parameters from asymmetric limit cycle data by a biased relay with hysteresis. The control synthesis is then performed through a solution of the Diophantine equation in the ring of proper and stable RQ-meromorphic functions. The methodology generates a class of generalized PI or PID controllers. The design method brings a scalar tuning parameter $m_{0}>0$ that can be adjusted by various strategies, the "equalization" method in Gorez and Klán (2000), Pekař et al. (2007) can be one of them. The methodology is illustrated by the example of higher order and dynamic. The program system for design, tuning and simulation is developed as well.

\section{ACKNOWLEDGMENTS}

This work was supported by the grant of Ministry of Education of the Czech Republic No. MSM 708835.

\section{REFERENCES}

Åström, K.J. and Hägglund, T. (1984). Automatic tuning of simple regulators with specification on phase and amplitude margins, Automatica, no.20, pp.645-651. de Arruda, G.H.M. and Barros, P.R. (2001). On a relay experiment for frequency response estimation, Proceedings of the European Control Conference, pp. 669-674.

Fliess, M., Marques, R., and Mounier, H. (2002). An extension of predictive control, PID regulators and Smith predictors to some linear delay systems, Int. J. of Control, vol. 75, No. 10, pp. $728-743$.

Gorez, R. and Klán, P. (2000). Nonmodel-based explicit design relations for PID controllers, Preprints of IFAC Workshop PID’00, pp. 141-146.

Kaya, I. and Atherton, D.P. (1999). A new PI-PD Smith predictor for control of processes with long death time. IFAC $14^{\text {th }}$ World Congress, pp. 283-287.

Kučera, V. (1993). Diophantine equations in control - A survey, Automatica, Vol. 29, pp. 1361-75.

Majhi, S. and Atherton, D.P. (1998). A new Smith predictor a controller for unstable and integrating processes with time delay, IEEE Conf. on CDC'98, pp. 1341-1345.

Pecharromán, R.R. and Pagola, F.L. (2000). Control design for PID controllers auto-tuning based on improved identification, Preprints of IFAC Workshop PID’00, pp. 89-94.

Pekař, L., Prokop, R., and Matušů, R. (2007). Algebraic control of unstable delayed first order systems using $R Q$-meromorphic functions, Proceedings of the 15th Mediterranean Conference on Control and Automation, Athens, Greece, [CD-ROM].

Pekař, L. and Prokop, R. (2009). Some observations about the RMS ring for delayed systems, Proc. $17^{\text {th }}$ Int. Conf. on Process Control '09, Slovakia, 28-36.

Prokop, R. and Corriou, J.P. (1997). Design and analysis of simple robust controllers, International Journal of Control, Vol. 66, No. 6, pp. 905-921.

Prokop, R., Husták, P., and Prokopová, Z. (2002). Simple robust controllers: Design, tuning and analysis, Preprints of $15^{\text {th }}$ IFAC World Congress.

Smith, O.J.M. (1957). Closer Control of Loops with Death Time. Chem. Eng. Prog. 53, p. 217.

Thyagarajan, T. and $\mathrm{Yu}$, Ch.Ch. (2002). Improved autotuning using shape factor from relay feedback, Preprints of IFAC World Congress.

Vidyasagar, M. (1985). Control system synthesis: a factorization approach, MIT Press, Cambridge, M.A.

Vítečková, M. and Víteček, A. (2004). Experimental identification by relay methods (in Czech), Automatizacia a informatizacia.

Vyhlídal, T. (2000). Anisochronic first order model and its application to internal model control, ASR '2000 Seminar.

Yu, Ch.Ch. (1999). Autotuning of PID Controllers, Springer, London.

Zítek, P. and Víteček, A. (1999). The design of control of subsystems with delays and nonlinearities (in Czech), CVUT publishing, Praha.

Zítek, P. and Kučera, V. (2003). Algebraic design of anisochronic controllers for time delay systems, International Journal of Control, Vol. 76, No. 16, pp. 905-921. 